\title{
CHARGE CONTRAST IMAGING IN VARIABLE PRESSURE SEM: CORRELATION, OPTIMISATION AND APPLICATION
}

\author{
Brendan J Griffin and Alexandra S. Suvorova
}

Centre for Microscopy and Microanalysis, The University of Western Australia, 35 Stirling Highway, Crawley, WA 6009

Charge contrast imaging (CCI), as initially reported ${ }^{1}$, revealed growth structure in hydrated aluminium hydroxide (gibbsite); data not attainable by any other electron, laser or light optical imaging technique. This new contrast result was achieved with a specific set of operating conditions of the variable pressure or environmental SEM. CCI have subsequently been reported from a range of poor and non-conductors. The detail of CCI has also been shown to correlate with cathodoluminescence in many natural minerals and synthesised materials ${ }^{2,3}$. The operating conditions for optimal contrast in CCI have been characterised for a range of previously reported responsive materials. CCI is found to be observable in a multi-dimensional envelope of VPSEM operating conditions. The more recently described techniques that demonstrate the benefits of reduction of positive ion fluxes above the sample have been found to extend to the 'CCI envelop', a confirmation of earlier studies (Doehne, pers. comm.).

New studies of the retardation of the primary electron landing energies, by measurement of DuaneHunt limits in EDS X-ray spectra, have been undertaken in parallel with the characterisation of CCI conditions. Specific CCI detail are found not to be observed when the landing energy of the primary electron beam is significantly retarded, ie when the sample is negatively charged (figure 1). This result is reproducible and consistent within a range of studied materials. The compositional control on charge balance, as observed in Duane-Hunt values across a range of materials (figure 2), matches the variations in conditions required for optimal CCI in these materials.

A parallel study has confirmed that the detail observed in CCI are not visible when samples are examined under very low accelerating voltage conditions in field emission SEM (FESEM), even when uncoated, under conventional high vacuum conditions. These data confirm the view that CCI is separate from the long-recognised voltage contrast imagery. Significantly, the surface of our 'CCI responsive' test samples have been observed to have a thin surface contaminant layer, in these FESEM images. These observations are consistent with the recently reported observation that CCI can be used to images defects below surficial deposits ${ }^{4}$.

The new data support the view that CCI is affected strongly by variations in sample conductivity at the micro or even 'nano' scale. CCI visibility through surface contaminant layers supports a view that induced field fluctuations from variations in trapped charge, and its decay, may carry the contrast data rather than direct or specific actual emissions.

\section{References}

1. B.J. Griffin Microsc. Microanal., 3 (s2) (1997), 1197.

2. E. Doehne, Scanning, 19(1997)75.

3. B.J. Griffin et al., Microsc Microanal 5(1999)278.

4. S.P.Galvin et al., J. Materials Science, 36(2001). 

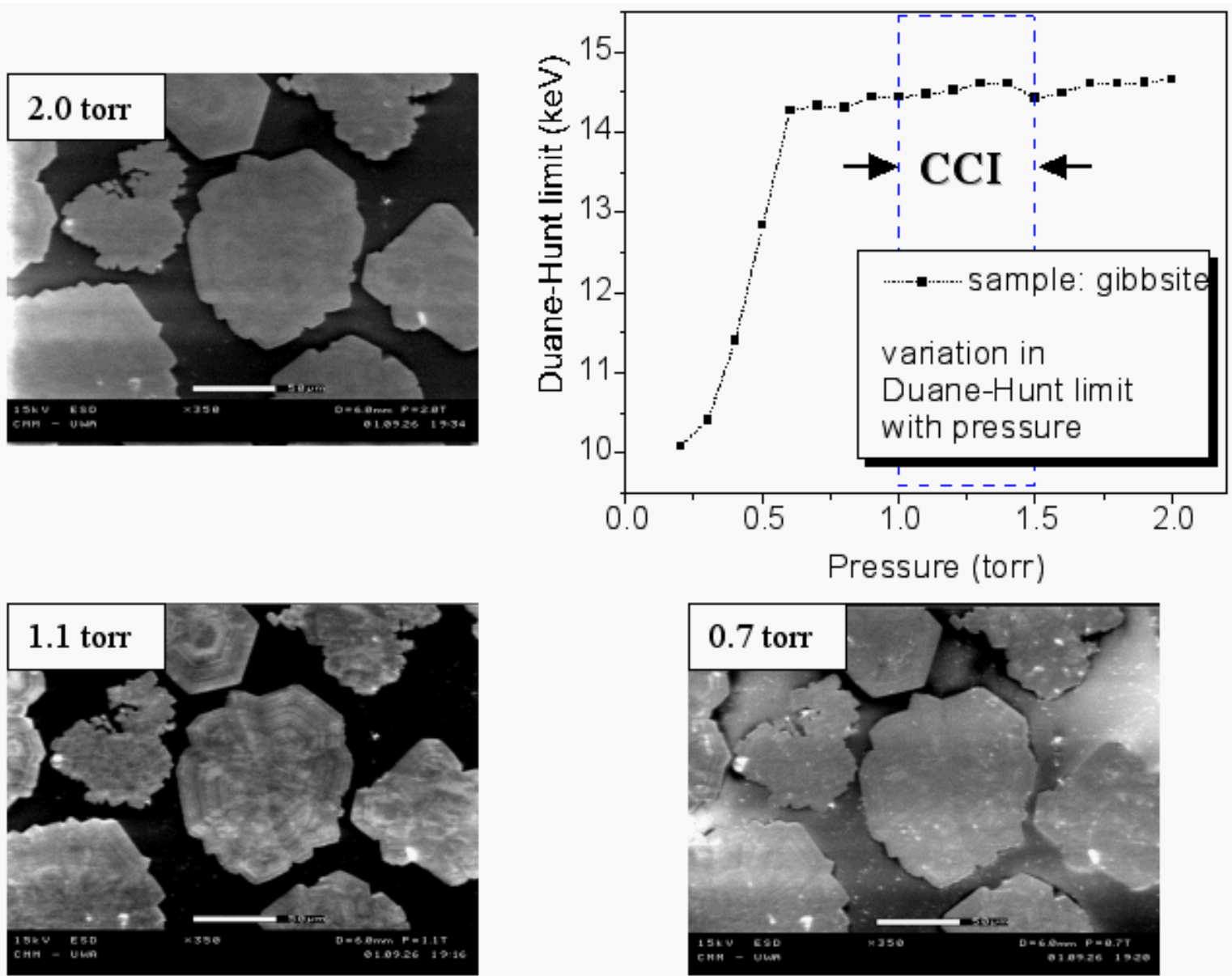

Figure 1: Comparison of the 'CCI envelope' and sample charging effects on the primary electron landing energy.

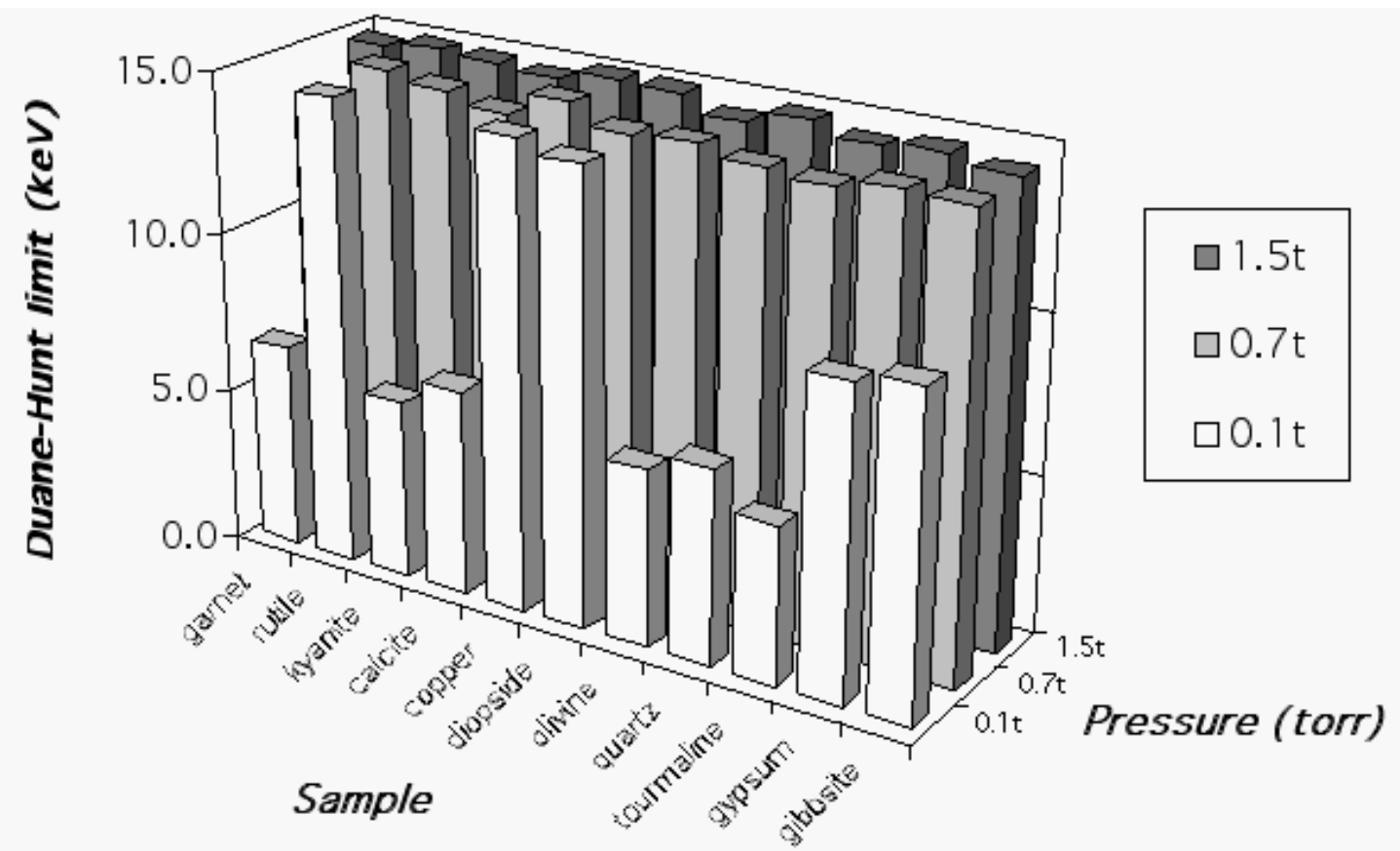

Figure 2: Effects of sample composition and and chamber gas pressure on the primary electron retardation in VPSEM. 